Hartwell, L. M. (2010). Impact of software design on on-line text reconstruction. System, 38(3), 370-378. http://www.sciencedirect.com/science/article/pii/S0346251X10000710.

Author's copy

\title{
Impact of software design on on-line text reconstruction
} Laura M. Hartwell

Service des Langues, Universite' Joseph Fourier, 38 rue Barthélémy de Laffemas, BP 2, 26901 Valence cedex 09, France

Abstract

This study examines the impact of software design on user practices concerning progression and performance while working on a text reconstruction activity that is preceded by an audio-visual presentation. The entries of participants during their first task of a science-based on-line activity aimed at French-speaking University students are analyzed. The entries of 97 students working on the first activity are described in detail, and then the impact of correctness on terminating the first activity of a larger population of 142 students is examined. Students of varying degrees of motivation and language knowledge appear to personalize the task. Users generally proceeded in a linear fashion, although the option of skipping from one sentence or one paragraph to another within the text is fully available. The immediate feedback after mistakes appears to encourage users to re-enter a new spelling or conjugation of the word or a new word. While high-frequency grammatical words were often entered, avoidance of more difficult words was also recurrent. The findings suggest that users do rely upon meaning as well as language knowledge when searching for answers.

There is a greater short-term retention of words in the first paragraph of the text and of text incorporated within the visual supports. While the use of dots representing letters is not relied upon by users, the immediate feedback appears to offer learners the opportunity to immediately review common mistakes such as homophones or word endings. This immediacy of feedback is a strength of computer learning that is often difficult to achieve in a classroom.

Keywords: Text reconstruction; Word placement; Visual supports; English for special purposes

\section{Introduction}

Within the realm of computer-assisted language learning, on-line learning is one of the most rapidly developing sectors. The on-line publication of learning activities goes hand-in-hand with the increasingly complex technological advances. One example of this phenomenon is Enigma Plus (Upjohn, 2007), an on-line listening and text reconstruction platform designed for learning English as a foreign language in a science-based university (See: http://elang. ujf-grenoble.fr/enigma/). This article examines the impact of software design on user entries during on-line study. The research hypothesis is that certain aspects of the software design, including word order, visual supports and design conception affect practice. For this study, the entries of 97 subjects working on the activity Reverse Osmosis of the platform were analyzed to determine language difficulties, the impact of word placement and the retention of text incorporated within the visual supports. Then, in order to determine if a series of linguistic difficulties was linked to the user ending the activity, the number of correct answers for a larger population of 142 users was studied.

2. Oral and visual computerized input

Exposure to comprehensible input has long been a central notion in language learning, notably in the theory of Natural Approach (Krashen and Terrell, 1983; Vandergrift, 2007). For oral comprehension, the characteristics of simplified oral documents used for language learning may include a slower-than-average rate of speech, clear articulation, high-frequency vocabulary and short phrases with a simplified syntax. The English document in the current study employed high-frequency words found in scientific discourse. The speech of the recording was well articulated and without hesitations, misspeaks, or background noise. 
Images are often touted as aiding in understanding oral documents (Chaudron, 1995; Cornaire and Germain, 1998; Rost, 1990; Ur and Wright, 2005). Visual supports offer a context for understanding, often offer traces of writing and may help in memorizing information. However, images or text within visual documents may also be a source of conflict or confusion when they do not match the oral document (De'cure', 1996; MacWilliam, 1986). Oral and visual documents are increasingly found together and require new skills for the language learner (Kress and Burn, 2005). The illustrations of this platform do not include images of the person speaking, but animated slides, including text. The oral and visual documents included in this study are in synchrony. The visual aids were not designed to conflict with the oral document, but to enhance comprehension.

\section{Background}

The reconstruction in the activity studied is done using a computerized skeleton of the text. It is an adaptation of John Higgins's Storyboard, which emanated from his program Rebuild, inspired by Tim John's Textbag in the early 1980s. Numerous other editions, in various languages, were created during the 1980s (Davies, 2007). The first Enigma platform was created at the same time as the textbook, later published as Minimum Competence in Scientific English (Blattes et al., 2003). In 2006, an improved version of the platform, that includes oral recordings and synchronized visual supports, was published on the university server as part of a foreign language self-learning policy. The current study examines user progression and error when using this new version of the program.

\section{Text reconstruction}

For this activity, the learner listens to a recording while observing a series of supporting slides. The oral document is the voice of a native British man speaking clearly and distinctly. The color slides present schematic graphics and keywords (cf. Fig. 1). The corresponding words appear on the screen as they are spoken. After the presentation, a skeleton of the text is automatically displayed on the screen. The first two letters of each word are given, and the other letters are represented by dots (cf. Fig. 2). For example, the word osmosis is represented as "os__ ". Using the option "easy mode", users see the first three letters of each word. Words that students are not expected to know, such as geographical locations or formulas, are spelled out completely within the skeleton. The user types a word in the window at the bottom of the screen and clicks on the mouse. The word then appears throughout the skeleton. If the word is not in the skeleton, a verbal or written message encourages the user to enter a new word.

\section{The present study}

\subsection{Participants}

The 142 participants were pursuing an undergraduate degree in science and technology in a French University, which is listed in the Shanghai list of universities. Second-year students at this institution are required to attend English class and hence to work on at least two texts of Enigma Plus. There is no obligation concerning the amount of time spent, the number of entries or the number of correct answers. Students can work at their own rhythm either at home or in the university language labs where a tutor is present. Students could work on-line at any time during the semester. The list of subjects is not ordered by level of difficulty or any other criteria. There were 12 possible subjects from which to choose. For their first exercise, 97 students chose the first title of the list, Reverse Osmosis. 


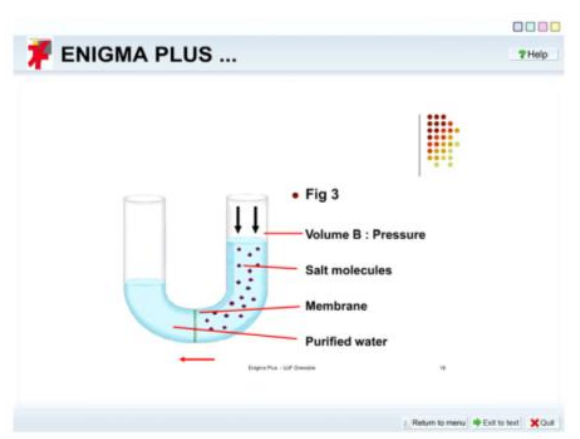

Fig. 1. Enigma plus e presentation slide.

\subsection{Methodology}

Each answer entered by the user was recorded automatically onto a server. The entries were then downloaded from the server for a quantitative and statistical analysis. The findings presented here are from an authentic self-learning context without interference by an observer. The research questions were related to how users proceeded when reconstructing the text. Notably, do users take advantage of the possibility offered by the computer to work anywhere on the text or do they proceed in a linear fashion? Did the presence of certain words on the visual supports have an impact on the user entering, or not entering, the given word? What were the common types of mistakes or errors? And finally, did a series of "non hits" occur before users stopped reconstructing the text?

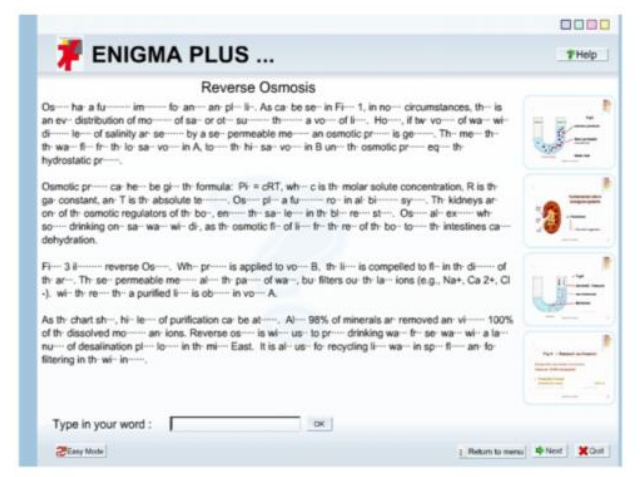

Fig. 2. Enigma plus e text screen.

\section{Results and discussion}

\subsection{First entry}

The first aspect examined is the order of word entry by the 97 students who chose to begin with the activity Reverse Osmosis. "Osmosis", present in the title and the first word of the text, was the first entry $(49.5 \%, n=48)$ for almost one-half of the subjects. The term osmotic ( $n=15)$ or the incorrect form osmotics $(n=1)$ was entered by a notable number of the participants $(16.5 \%, n=16)$. During the initial audio-visual presentation, the term osmosis is repeated verbally five times, but shown on the screen only in the title. Osmotic is repeated four times verbally and shown three times on the screen. However, the word osmotic is also incorporated into the skeleton text; there is therefore no reason for the user to enter it. Although osmotic is not appropriate in the rest of the text, sixteen users either copied it from the skeleton or remembered the morphology from the presentation.

Sixteen users (22.4\%) entered only the missing letters of osmosis or a version of osmosis, omitting the first "o" and " $\mathrm{s}$ " which are not represented by dots. The errors varied, but "mosis" was the most frequent form $(n=14)$. Osmosis was the second entry of two-thirds of this subgroup (62.5\%, $n=10)$ Two users experimented with other answers, for example entering a string of words: "osmosis has a fundamental importance for animal a" (sic). One user having 
entered "mose", wrote out the French word osmose, before finding osmosis. Finally, one user entered "motic", then osmotic and then entered 48 other answers (including osmotic twice and once osmotic pressure) before finding osmosis.

Only one user had significant difficulties during his first study session. His first entry was "ng" followed by "otis". He then wrote "osmotis" and osmotic before entering osmosis. Twice in a row he wrote "anithing" instead of anything, demonstrating spelling limitations. During the following days, he continued to work on different exercises and showed clear improvement. The overall speed of adaptation to the program demonstrates that users were able to understand its functioning without relying on the instructions available by clicking on the "help" button in the top right-hand corner of the screen. This adaptation by trial and error was confirmed by a user whose first entry was test, a word not present in the text.

Other users attempted to enter the word osmosis, but had difficulties with the spelling. Among these attempts are "osmotique", "osmolarity", and the French word osmose $(n=2)$. All the participants found osmosis at one point or another of the activity. However, the number of mistakes, immediately after the audio-visual presentation in which osmosis appears on the screen once and is repeated orally four times, suggests that the presentation is insufficient for the acquisition of the correct spelling of a word. The reactivation of the word in written form appears to be justified. In other words, one does not learn by osmosis alone (Mendelsohn, 1994).

Finally, $11.2 \%(n=11)$ of the users entered an answer that was related to the text, but not osmosis or a related form. Half of this sub-group entered has $(n=5)$, the second word of the text and which was undoubtedly easier to spell than osmosis. The other entries were allows, animal, wine and industry. Wine and industry are the last two words of the text and are written on the last slide of the presentation. This would indicate that these two users paid particular attention to the presentation and were aided by the "recency effect", the phenomenon that last items are remembered more easily as demonstrated by Glanzer and Cunitz in their 1966 article Two storage mechanisms in free recall (Fenouillet, 2003). The results show that the vast majority of users begin at the beginning of the text. There is automatic feedback after each word entry. The feedback simply notifies the user if the entry is correct or if not, prompts them to enter a new answer. Users do take advantage of this opportunity to enter a new response.

\subsection{Second and third entries}

An analysis of the second and third entries confirms the practice of working in a linear fashion. Osmosis was the first correct answer for two-thirds of the users $(63.9 \%, n=62)$. Two-thirds of this sub-group $(n=41,66.1 \%)$ continued with the second word of the text, has. Another $8 \%$ of the subjects $(n=5)$ made an error of conjugation and entered either had $(n=1)$ or have $(n=4)$. The remaining portion of this sub-group $(16.5 \%, n=16)$ decided to work on other words of the text, notably fundamental (third word of the text, $n=6$ ) and figure $\left(12^{\text {th }}\right.$ word of the text, $\left.n=3\right)$. This means that almost half the population $(40 \%, n=47)$ progressed in a linear fashion for the first two words (cf. Table 1). 
Table 1

Second entry (after osmosis) and third entry (after osmosis has).

\begin{tabular}{lcc}
\hline Word & Osmosis & Osmosis has \\
\hline A & 0 & 1 \\
And & 0 & 2 \\
Animal & 0 & 1 \\
Figure & 3 & 4 \\
For & 0 & 9 \\
Full & 1 & 4 \\
Function & 0 & 4 \\
Fund & 0 & 1 \\
Fundamental & 3 & 14 \\
Fundamentale (sic) & 0 & 1 \\
Funny & 1 & 0 \\
Futur (sic) & 1 & 0 \\
Importance & 1 & 0 \\
Important & 2 & 3 \\
Improve & 1 & 0 \\
Pression (sic) & 1 & 0 \\
Temperature & 1 & 0 \\
The & 1 & 0 \\
There & 0 & 1 \\
When & 1 & 0 \\
Total & 17 & 45 \\
\hline
\end{tabular}

There was slightly more variation for the third word entered. The sub-group of participants whose first two correct entries were osmosis has $(n=45)$ followed with a range of twelve different entries (cf. Table 1). Fourteen of these participants continued with a correct form of the text's third word fundamental. The first two letters of each word are shown on the screen, so fundamental is represented as " $f u$ ". It is also the only word of the text beginning with the letters "fu", so one can suppose that entries beginning with "fu" are an attempt to find that word. Among these attempts are full $(n=4)$, function $(n=4)$, fund $(n=1)$ and fundamentale $(n=1)$. If they were searching to find the word fundamental, then a quarter of the population $(24.7 \%, n=24)$ proceeded in a linear fashion for the first three words. Three users wrote the adjective important which is undoubtedly an attempt to find the fourth word of the text importance. The fact that they had not yet found the adjective fundamental is likely to have influenced their choice to enter an adjective instead of a noun.

The sub-group whose first correct answer was osmosis, but who did not type has $(n=17)$ entered a selection of twelve different words. Like the sub-group which had entered osmosis has, this sub-group made attempts to find a word beginning with "fu", including the errors: full $(n=5)$, function $(n=4)$, fund $(n=1)$, funny $(n=1)$, fundamentale $(n=1)$, plus the French word futur $(n=1)$. By enlarging the analysis to the complete collection of data, we find that significant errors were made in the attempt to find the missing word including: full and its variations fullness and the misspelling "fullther" $(n=19)$, function and its variations functio(n)nal and functionality $(n=34)$, fund $(n=3)$, funny $(n=7)$, futur $(n=1)$ and fundamentale $(n=2)$. Four users attempted to find the fourth word importance $(n=1)$, of which three entered the adjective important $(n=2)$ and a verb improve $(n=1)$. This implies that users are following different types of achievement strategies. While some users have a general notion of meaning (fundamentale, functionality), others are searching linguistic clues (fullness, funny). It is interesting to note that a majority of the users do not rely upon the visual aid of dots representing letters to estimate the length of the word to be entered. Displaying the first two letters of each word is a design choice that does in fact aid users in finding an answer. However, a series of dots representing the total number of letters does not offer an aid that engages users.

\subsection{Overall linear progression}

We have seen that the majority of users begin at the beginning of the text, but by the third correct answer only one-quarter progress in a strictly linear fashion. In order to examine the overall progression, the results for the four paragraphs of the text were analyzed. Words that appeared in two or more paragraphs were not included. This offers a global overview of the 
text. Table 2 illustrates that users are significantly more likely to find words located in the first paragraph of the text. Setting aside repeated words a $t$-test was used to compare the mean of the frequency that users found the 68 words from the four paragraphs of the skeleton. Compared to the frequency of words found in the first paragraph, a $t$-test determined that significantly fewer words were found in the three following paragraphs $(p=0.001)$. Furthermore, while the minimum number of correct entries for a given word in the first paragraph was 48, in the following three paragraphs, the minimum fell as low as only 10 users finding the word constant. It appears that even if users do not progress strictly word by word, significantly greater attention is paid to the beginning of the text.

This phenomenon of progressing from beginning to end even in a virtual world where there are no limits to navigation was also confirmed by user choice of subject. The titles of subjects of Enigma Plus are presented in two columns of a dozen independent subjects without any reference to difficulty or ideal order. This study showed that $42.3 \%$ of the 142 users "clicked" the first subject for their first activity (osmosis) and $28.2 \%$ clicked the second title (Mercury) for their second activity. There was little "virtual detour" to other topics. When designing online or computerized programs and activities, it appears important to keep in mind that users will spend more time and attention on items at the beginning than at the end.

\subsection{Impact of visual supports}

The presence of a given word within the visual supports also appears to impact user performance. The visual supports are automatically displayed when the user clicks on the title of choice and the oral document begins. To examine the impact of these supports, the seven words that appeared on the presentation screens and are included only once in the skeleton were compared to the 63 terms that were not in the visual supports and included only once in the text to reconstruct. The seven words appearing on the visual supports are spread over the first paragraph (even), the second (role, biological, systems) and the fourth (space, wine and industry). Table 3 displays the results of the comparison. First, it can be noted that the majority of the users did not encounter difficulty with any of the words that appeared on the screens. The word least often found was space, but it was still found by 62 users. In contrast, a majority of the words $(n=31)$ that did not appear on the screens were found by 62 users or less. A $t$-test comparing the mean of words not shown to those shown reveals a probability of $p=0.001$. So, displaying words on the visual supports is a second significant factor.

This finding suggests that for a better retention of vocabulary, a content word should be shown on the screen. As the print on the thumbnails of the presentation slide is too small to be read on the text reconstruction screen, we can note that users apparently retain the words initially seen during the presentation section. For example, the expression space flights is spoken, while in the visual support the expression space travel is in written form. A majority of the users $(62.9 \%, n=61)$ found the term space, but only 36 found the following word flights. The word flights is probably also less well known or transparent to the subject population than the term travel. However, this does not mean that the oral document must repeat the words present within the visual supports - certainly this is not the case in authentic scientific presentations.

Furthermore, as we saw with the example of "osmosis", simply appearing on the visual support or being included in the oral document is not sufficient for retention. The redundancy factor via the on-line skeleton appears to be necessary.

\subsection{Preference for grammatical words}

In order to determine if content or lexical nouns were entered more often than grammatical words, the mean of each type of word was calculated. The 16 grammar words listed only once in the skeleton were entered on an average of 75.56 (standard deviation $=19.06$ ). This category includes words such as has, this or where, which can be found in the four paragraphs, but are uncommon on the visual supports. Users had the greatest difficulty with throughout $(n=48)$ and the link word hence $(n=42)$. Their overall average is much higher than the general mean of 64.1 and the difference $p=0.014$ is also significant (cf. Table 4). 
Table 2

$t$-Test of word frequency according to placement.

\begin{tabular}{lllllll}
\hline Paragraph & Number of words & Minimum & Maximum & Mean & Standard deviation & $t$-Test \\
\hline 1 & 22 & 48 & 97 & 80.23 & 13.70 & $p=0.001$ \\
2 & 22 & 10 & 92 & 57.18 & 21.53 & $p=0.072$ \\
3 & 10 & 40 & 87 & 56.00 & 13.16 & $p=0.041$ \\
4 & 14 & 36 & 87 & 55.43 & 15.06 & $p=0.024$ \\
Total & 68 & 10 & 97 & 64.10 & 19.95 \\
\hline
\end{tabular}

Table 3

$t$-Test of impact of written trace on performance of words cited once in skeleton.

\begin{tabular}{lllllll}
\hline Display of word & Number of words & Minimum & Maximum & Mean & Standard deviation & $t$-Test \\
\hline On screen & 7 & 62 & 95 & 75.14 & 12.17 & $p=0.028$ \\
Not on screen & 61 & 10 & 97 & 63.08 & 20.06 & $p=0.319$ \\
Total & 68 & 10 & 97 & 64.10 & 19.95 \\
\hline
\end{tabular}

In comparison, content or lexical nouns were entered on an average of 64.32 , which was close to the general average. They were evenly distributed among the four paragraphs, but words in the first half of the skeleton were found more often $(77.6 \%)$ than the words in the second half of the skeleton $(52.83 \%)$. Five of the 22 lexical or content nouns were also present on the visual supports, including the terms role, systems, and space discussed later. The mean entry for these five words is 71.8 , which is higher than the other lexical words, but lower than the average of on-screen words (75.14). These findings are to be read with caution as the sub-group is limited to five words. A $t$-test comparison of grammar words compared to the average of content nouns reveals a significant probability of $p=0.012$. So, students were more motivated to enter frequent grammar words instead of searching for nouns related to scientific vocabulary, such as substance $(n=64)$, passage $(n=49)$ or waste $(n=36)$. It is probable that users attempted to enter common grammatical words first, perhaps to fill gaps and to make finding difficult lexical words easier to find. However, while this strategy may have been constructive, it was insufficient in that, overall, lexical nouns were found less often than the common grammatical words.

\subsection{Language mistakes}

Several mistakes related to language were recurrent and merit mention. One mistake was an overuse of the plural form: 25 participants entered the plural form animals before finding the sixth word of the text in its singular form animal $(n=81)$. The interference of the first language was also evident. For example, 41 users wrote the French word "gaz" instead of the English word gas $(n=60)$, and 14 subjects wrote liquid with an "e" producing the French equivalent "liquide", another 10 participants added an "e" to plant, forming the French word "plante". Once again, this demonstrates that users do not rely upon the quantity of dots to evaluate if an "s" or an "e" is necessary. However, the design conception of the immediate opportunity to enter a new answer does appear to be a positive one. For example, of the 41 users entering the French word gaz, 31 followed up by entering "gas". Five of these users reentered gaz before finding "gas". However, five users did not re-enter a three-letter answer beginning with "ga". So, while this design conception offers users the possibility to review spelling, the general feedback is not always sufficient enough for users to negotiate a correct answer.

As with the re-entry of the French word gaz, users held fast to their initial misperception. For example, twenty-nine participants wrote see instead of its homophone sea $(n=51)$. Of these users, eleven of them re-entered the word see at least a second time. It appears that users are convinced that there was a technical or design error. For example, a student complained that an apostrophe within the visual support was incorrectly placed. She did not know that 
when a noun is in its plural form the apostrophe demonstrating possession is placed to the right of the letter "s".

\begin{tabular}{|c|c|c|c|c|c|c|}
\hline Type of word & Number of words & Minimum & Maximum & Mean & Standard deviation & $t$-Test \\
\hline Grammar & 16 & 42 & 97 & 75.56 & 19.06 & $p=0.014$ \\
\hline Lexical noun & 22 & 36 & 92 & 64.32 & 16.30 & $p=0.475$ \\
\hline Total of all words & 68 & 10 & 97 & 64.10 & 19.95 & \\
\hline
\end{tabular}

Table 5

Total number of correct answers and correct answers during last 10 entries.

\begin{tabular}{lccccr}
\hline Correct answers & $1-50$ words & $51-75$ words & $76-100$ words & $100+$ words & Total \\
\hline $0-3$ /last 10 & $8(20.5 \%)$ & $9(23.1 \%)$ & $14(35.9 \%)$ & $8(20.5 \%)$ & $39(27.5 \%)$ \\
$4-5 /$ last 10 & $11(31.4 \%)$ & $15(42.9 \%)$ & $6(17.1 \%)$ & $3(8.6 \%)$ & $35(24.6 \%)$ \\
6-7/last 10 & $9(25.7 \%)$ & $8(22.9 \%)$ & $9(25.7 \%)$ & $9(25.7 \%)$ & $35(24.6 \%)$ \\
$8+$ /last 10 & $5(14.2 \%)$ & $4(12 \%)$ & $12(36.4 \%)$ & $12(36.4 \%)$ & $33(23.3 \%)$ \\
Total & $33(23.3 \%)$ & $36(25.4 \%)$ & $41(28.9 \%)$ & $32(22.5 \%)$ & $142(100 \%)$ \\
\hline
\end{tabular}

6.7. Ending the task

We saw previously that users are more likely to enter frequent grammar words than sciencerelated content nouns.

In order to determine if a series of linguistic difficulties, or in other words a series of "nonhits", was linked to the user ending the activity, the number of correct answers for the total population of 142 users was counted. In Table 5, the total number of correct answers was compared with the number of correct answers during the last 10 answers.

The regular distribution of the results implies a wide range of capacity and motivation to reconstruct the text.

Slightly over one quarter of the users had only three or fewer correct answers during the last 10 entries. Members of this sub-group are found in all four categories of total amount of correct answers; the categories being from 1-50 words to 100+ words. Users finding between 51 and 75 correct answers appeared to lose motivation when entering a series of four or five correct words out of 10 (42.9\%). However, users who came closer to reconstructing the text by entering more than 76 correct entries ended the activity while continuing to hit upon a greater number of correct answers (36.4\%). Hence, the language knowledge necessary to reconstruct the text appears to have limited impact on the overall user decisions to end the activity. The results show a wide range of quantities of correct answers, even just before ending the exercise.

7. Conclusion

This study examines the impact of software design on student practices concerning progression and performance while working on a text reconstruction activity. This task does not resemble real-life language use such as listening to a conference presenter or class note taking. It does offer the opportunity to slow down the listening process and to negotiate meaning by permitting multiple attempts to identify words. The findings suggest that users do rely upon meaning as well as language knowledge or, in other words, content as well as form when searching for answers.

The preference for the first paragraph of the text and the impact of text incorporated into the visual supports are important considerations when designing on-line language learning activities. The fact that users enter words that were previously displayed on the screen would support the hypothesis that redundancy helps retention.

Users of varying degrees of motivation and language knowledge appear to personalize the task. This hypothesis is supported by the wide range of standard deviation for the entry of content words. While the use of dots representing letters is not relied upon by users, the immediate feedback appears to offer learners the opportunity to immediately review common mistakes such as homophones or word endings. This immediacy of feedback is a strength of computer learning that is more difficult to achieve in a classroom. The observation that users frequently do not enter "difficult" nouns such as dehydration or formula suggests that special attention should be given to these words if students are to benefit from the inclusion of this 
category of words in the text. Perhaps the volume of words to be found inhibits concentration on the targeted vocabulary.

One limitation of this study is that the use of the option of listening to a given section was not examined. This option is an important element of the design, but does not appear on the computer readouts of student answers. Further studies could be conducted that evaluate the impact of this option. However, the effect of an observer recording practice should then also be taken into account.

Another limitation of this study is that long-term learning is not evaluated. Further studies are needed to assess the impact of the program both on students studying the vocabulary and concepts in class or users who are independently using the program without an institutional context. The impact may include effects on listening comprehension, fluency when speaking, integration of visual supports during oral presentations, retention of target vocabulary, conjugation or first-language influence on spelling.

\section{References}

Blattes, S., Jans, V., Upjohn, J., 2003. Minimum Competence in Scientific English. EDP Sciences, Les Ulis, France.

Chaudron, C., 1995. Academic listening. In: Mendelsohn, D., Rubin, J. (Eds.), A Guide for the Teaching of Second Language Listening. Dominie Press, San Diego, CA, pp. 74-96.

Cornaire, C., Germain, C., 1998. La Compréhension Orale. Collection Didactique des langues étrangères/CLE International, Paris.

Davies, G., 2007. Total Cloze Text Reconstruction Programs: a Brief History. www.ict4lt.org/en/FWTHistory.doc (accessed 02.09.08).

Décuré, N., 1996. Images and Imagination, vol. 7. Les Après-midi de LAIRDIL. $<$ http://www.lairdil.org/htm/publi.php?idmembre=1> (accessed 06.06.06).

Fenouillet, F., 2003. Motivation, Mémoire and Pédagogie. L'Harmattan/Editions Savoir and Formation, Paris.

Krashen, S., Terrell, T., 1983. The Natural Approach: Language Acquisition in the Classroom. Pergramon Press, Oxford.

Kress, G., Burn, A., 2005. Pictures from a rocket: English and the semiotic take. English Teaching: Practice and Critique 4 (1), 95e105. http:// education.waitkate.ac.nz/research/files/etpc/2004v4n1art7.pdf (accessed 06.10.07).

MacWilliam, I., 1986. Video and language comprehension. ELT Journal 40 (2), 131-135. Mendelsohn, D., 1994. Learning to Listen: A Strategy Based Approach for the Second Language Learner. Dominie Press, Inc., San Diego, CA.

Rost, M., 1990. Listening in Language Learning. Longman, London.

Upjohn, J., 2007. Engima Plus. http://elang.ujf-grenoble.fr/enigma/ (accessed on 02.09.08).

Ur, P., Wright, A., 2005. Five-minute Activities: A Resource Book of Short Activities.

Cambridge Handbooks for Language Teachers. Cambridge University Press, Cambridge.

Vandergrift, L., 2007. Recent developments in second and foreign language listening comprehension research. Language Teaching 40 (3), 